\title{
Proyecto de comercialización de la gamitana en Lima,
}

\section{Perú}

\begin{abstract}
RESUMEN
La gamitana es un pez originario de las cuencas de los ríos Amazonas y Orinoco, con una demanda creciente en el Perú, especialmente en los supermercados y restaurantes de comida típica de la selva, con una demanda aproximada de 600 kilos/semana, debido a la calidad del producto, por sus propiedades organolépticas homogéneas, su estricto control de calidad, sus certificaciones y auditoria del producto.

Los objetivos de este estudio son redefinir la estrategia y rediseñar la cadena de suministro de Puñizas SAC, empresa familiar ubicada en Junín dedicada a la producción y distribución de la gamitana a los supermercados y restaurantes de comida de la selva en Lima, para añadir valor a los clientes enfocado en el flujo del producto (la gamitana).
\end{abstract}

Palabras-claves: Gamitana; acuicultura; comercialización.

A COMMERCIALIZATION PROJECT FOR GaMitana IN Lima, Peru

\section{ABSTRACT}

The gamitana is a fish native to the Amazon and Orinoco River basin, with a growing demand in Peru, especially in supermarkets and restaurants specializing in traditional Amazonian jungle cuisine, with an estimated demand of approximately 600 kilos per week, due to the product's quality, homogeneous organoleptic properties, strict quality control, certifications and product auditing.

The objectives of this study are to redefine the strategy and redesign the supply chain of Puñizas SAC, a familyrun company located in Junín dedicated to gamitana production and distribution to supermarkets and Amazonian restaurants in Lima, in order to add value to clients focused on product (gamitana) flow.

Keywords: Gamitana; aquaculture; commercialization.

\section{INTRODUCCIÓN}

La acuicultura es una actividad significativa para el desarrollo de muchas familias en la selva del Perú (Instituto Nacional de Estadística e Informática, 2018). En la selva se cultivan especies propias como la gamitana, el paco, el paiche y el sábalo cola roja con una demanda creciente (Ministerio de la Producción, 2013) (Ministerio de la Producción, 2018); pero la mayor concentración acuícola está en el cultivo de los camarones peneidos (Fenucci, 1988), la concha u ostión abanico, la trucha arco iris y la tilapia (Martinez , Chávez y Olvera, 1988) (Ministerio de la Producción, 2018).

La gamitana es una especie con un consumo creciente en la ciudad de Lima; en especial, en las cadenas de supermercados y en los restaurantes de comida típica de la selva (Ministerio de la Producción, 2013). A pesar de la demanda atractiva para los pequeños productores, el proceso de distribución del producto fresco presenta retos importantes para operar de manera eficiente y efectiva (Ministerio de Transportes y Comunicaciones, 2015) (Curonisy y Pastén, 2016).

La cadena de distribución de los productos de la selva peruana a la costa tiene una distancia aproximada de 350 kilómetros. La selva central tiene una altitud de 1800 m.s.n.m, con un clima propicio para la producción de alimentos como frutas (granadilla, naranja, plátano), café (Ministerio de Comercio y Turismo, 2016), cacao (Ministerio de Comercio y Turismo, 2016), pescados, entre otros; estos productos cruzan la cordillera de los Andes, cuya altitud máxima es de 4818 m.s.n.m, recorre longitudinalmente la sierra y bajan a la costa peruana (Curonisy y Pastén, 2016). El recorrido, desde la selva hasta la costa, tiene un tiempo aproximado de nueve horas en condiciones favorables (Ministerio de Transportes y Comunicaciones, 2015) (Ministerio de Transportes y Comunicaciones, 2018).

1 Máster en Gestión de Agronegocios y Alimentos de la Universidad del Pacífico. Consultora independiente.

E-mail: y.curonisy@up.edu.pe

2 Máster en Gestión de Agronegocios y Alimentos de la Universidad del Pacífico, Decano de la Facultad de Ciencias Agrarias y Ambientales de la Universidad Católica Sedes Sapientiae. E-mail: jpasten@ucss.edu.pe

3 Doctor en Gestión de Empresas de la UNMSM, Vicedecano de Ingeniería Empresarial de la Universidad del Pacífico.

E-mail: m.chong@up.edu.pe 
La Carretera Central es la vía de acceso principal, principalmente de doble sentido (una vía de ida y una de regreso), se tiene una velocidad promedio de 40 kilómetros/hora (Ministerio de Transportes y Comunicaciones, 2018) y los productos están expuestos a los diferentes cambios de condiciones climáticas como la humedad, presión y temperatura. Entre los meses de enero a marzo, la distribución se dificulta por las inundaciones y los deslizamientos de lodo y piedras de los cerros (huaycos) y en muchos casos se pierde toda la carga. Las vías alternativas son por Canta, Huaraz y Cañete (Ministerio de Transportes y Comunicaciones, 2018), pero el tiempo del recorrido aumenta entre tres a seis horas.

El flujo de los productos, desde los productores a los consumidores finales, tiene una red de distribución caracterizada por los sobrecostos logísticos (Ministerio de Comercio y Turismo, 2016), los cuales, impactan directamente en la competitividad de los productos de la región (Atún, Lozano, Alarcón, Granados y Guarneros, 2010) (Ministerio de Transportes y Comunicaciones, 2015) (CAF Banco de Desarrollo de América Latina, 2017); a pesar del mejoramiento de la infraestructura de transporte intermodal (terrestre, marítimo, fluvial y aéreo) y las instalaciones logísticas (centros, parques y clústeres logísticos) (Ministerio de Transportes y Comunicaciones, 2015) (Ministerio de Transportes y Comunicaciones, 2018).

Los datos de este proyecto están basados en la encuesta del "Plan de negocio para la comercialización de gamitana en supermercados y restaurantes de Lima" (Curonisy y Pastén, 2016), en los estudios de la gamitana de los organismos gubernamentales (Instituto Tecnológico Pesquero del Perú , 2009) (Ministerio de la Producción, 2013) (Ministerio de la Producción, 2017) y el proyecto Gami Selva de la empresa Puñizas SAC.

Puñizas SAC es una empresa familiar ubicada en el departamento de Junín, tiene diez estanques y una capacidad de producción de dos toneladas mensuales de gamitana. La distribución de los 600 kilos/semana se realiza en los supermercados DC12 , supermercados DC-10 y en restaurantes de comida de la selva en la ciudad de Lima. Los supermercados DC-12 demandan 150 kilos/semana en sus dieciocho locales. Los supermercados DC10 demandan 350 kilos/semana en sus diez locales (se han usado los nombres de DC-12 y DC-10 por solicitud de los establecimientos). Los nueve restaurantes de comida típica de la selva demandan unos 150 kilos/semana (Curonisy y Pastén, 2016).
La sostenibilidad es un factor importante en este proyecto considerando su valoración de los consumidores de productos marinos, dejando en un segundo nivel la marca y el precio (Global Scan, 2015) (Global Scan , 2016). La confianza en las instituciones, en este caso con las entidades de la cadena de suministro, genera nuevas transacciones económicas, sociales y emocionales (Global Scan , 2016).

El estudio tiene como objetivos redefinir la estrategia y el rediseñar su cadena de suministro para añadir valor a los clientes enfocado en el flujo del producto (Perez-Franco, Sheffi y Caplice, 2016), concentrando toda la producción en el mercado limeño por sus precios atractivos y su creciente demanda. El horizonte de tiempo calculado es de 10 años con un crecimiento de ventas de un 15\% anual. El proyecto, tendrá un ingreso por ventas de S/. 145292 en el año primer año; con una proyección a S/. 2241758 en el décimo año (US\$ 750000 Dólares Americanos) y con un COK del $14 \%$ genera un VANE de S/. 258865 y un TIRE de 21,69\%.

\section{MARCO TEÓRICO}

El consumo de pescado en el Perú tiene un crecimiento de $2.4 \%$ anual, con un consumo per cápita de 21.8 kilos (Sociedad Nacional de Pesquería, 2014) y un gasto familiar del $7 \%$ de las canasta familiar en el año 2017 (Ministerio de la Producción, 2018). El sector pesquero creció $9.5 \%$ en el año 2017, en términos de volumen de extracción y la venta interna de recursos hidrobiológicos (Instituto Nacional de Estadística e Informática, 2018) (Ministerio de la Producción, 2018).

La acuicultura es una actividad económica rentable para los pobladores de la cuenca del río Amazonas, ecológica en su proceso productivo y nutricionalmente beneficiosa para los consumidores (Ministerio de la Producción, 2012) (Ministerio de la Producción, 2013) (Campos, 2015). En el 2015, la cosecha total de recursos hidrobiológicos procedentes de la acuicultura fue de 90976 toneladas, la extracción de las especies amazónicas fue de 36 750 toneladas; de las cuales 299 toneladas fueron de gamitana (Ministerio de la Producción, 2016).

La gamitana, Colossoma macropomum, es un pez originario de las cuencas de los ríos Amazonas y Orinoco; se cultiva en Bolivia, Brasil, Colombia, Panamá, Perú y Venezuela. En el Perú se cultiva en Cusco, Loreto, Madre de Dios, San Martín y Ucayali (Campos, 2015) (Ministerio de la Producción, 2017) 
(Ministerio de la Producción, 2018). El tamaño comercial de la gamitana está entre 25 y 30 centímetros, con un peso aproximado de un kilo. Una de las ventajas de la gamitana es su atractiva tasa de crecimiento del $42 \%$, comparado con el $24 \%$ de otras especies como el boquichico, el paco y el paiche (Instituto Tecnológico Pesquero del Perú , 2009) (Ministerio de la Producción , 2011) (Ministerio de la Producción, 2017).

El proyecto Gami Selva se inicia para satisfacer la demanda de gamitana fresca, con características organolépticas homogéneas y con un estricto control de la calidad (análisis microbiológico, análisis químico), las certificaciones de inspección y auditoría del producto final (Curonisy y Pastén, 2016). El proyecto tiene como público objetivo los supermercados y restaurantes de Lima aplicando las técnicas de reproducción y cultivo establecidas por los clientes y las entidades gubernamentales (Martinez, Chávez y Olvera, 1988) (Ministerio de la Producción, 2011) (Ministerio de la Producción, 2013).

El panorama descrito muestra una interconexión entre varias entidades con complejas relaciones, formales e informales, en una cadena de abastecimiento desde el productor hasta el consumidor final (Chopra, 2013). La administración de la cadena de abastecimiento, en este caso alimenticia, es un término de inicios de los años 80 , con una amplia difusión desde la entrevista en el Financial Times de Keith Oliver para describir los silos funcionales en una organización (marketing, operaciones, recursos humanos, finanzas) (Oliver y Webber, 1982) con énfasis en el suministro de los materiales y el alineamiento de la logística a la estrategia de las empresas (Heckmann, Shorten y Engel, 2003). La integración de las entidades, coordinación entre los flujos, comunicación de los procesos de negocio y la confianza en la cadena de suministro repercute en la productividad de los stakeholders (Wheelen y Hunger, 2013), la generación de valor en toda la cadena (Harrison y Van Hoek, 2011) (Chopra, 2013) y la satisfacción de los clientes (Krajewski, Ritzman y Malhotra, 2013).

La coordinación en un producto perecedero con una rápida alteración de sus condiciones desde la extracción hasta su consumo requiere complejos niveles de integración, cooperación, comunicación y confianza entre el productor y clientes (Bijman, y otros, 2010) (Chopra, 2013). El alineamiento de la logística con la estrategia se relaciona en la propuesta de valor o la relación entre la coherencia del ambiente externo e interno (Oliver y Webber, 1982)
(Chopra, 2013); la formulación de la estrategia relacionada con los objetivos a largo plazo; la implementación de las estrategias y sus planes funcionales (David, 2013) y la evaluación del desempeño bajo los planes transversales de responsabilidad social, sostenibilidad (Marine Stewardship Council, 2016) y ética.

Las prioridades competitivas de las organizaciones (costo, calidad, precio y tiempo) tienen en el plan de operaciones un instrumento de alineación, entre sus capacidades y sus recursos con la estrategia corporativa, evitando su desenfoque con una coherencia interna y externa entre cada una de las áreas (Chase, Jacobs y Alquilano, 2014). El plan funcional de operaciones se ve afectado por las acciones de los actores de ventas, marketing y finanzas en situaciones de ambientes cambiante, lo que obliga a ajustarlo frecuentemente (David, 2013). En el campo de la logística se ven afectados los planeamientos de compras en sus tiempos y costos. Esta situación debe considerar la consistencia interna (compatibilidad, coherencia y sinergia) (Perez-Franco, Sheffi y Caplice, 2016) en la estrategia de la empresa.

En este contexto es importante realizar pronósticos de demanda eficientes, ya que conforman la base para toda planeación de la cadena de suministro. Para ello, las empresas deben estar al tanto de factores como la demanda pasada, tiempo de reabastecimiento del producto, publicidad esperada, descuentos de precios planeados, estado de la economía y acciones que los competidores han realizado. El proceso de pronosticar es clave para toda decisión de diseño y planeación tomada en una empresa o en una cadena de suministro. Un fenómeno reciente son los pronósticos colaborativos que incrementan la precisión y permite la maximización del desempeño de la cadena de suministro (Chopra, 2013).

Los cambios en el mercado obligan a repensar la estrategia de la cadena de suministro considerando la velocidad del mercado (Perez-Franco, Sheffi y Caplice, 2016). Enfrentar estos cambios requiere de un enfoque innovador sobre los procesos de desarrollo de proyectos complejos con una orientación hacia el cliente con mayor agilidad en el seguimiento de las estrategias y debe evitar las reglas duras del enfoque tradicional; para afrontar las necesidades del mercado y resolver sus problemas con el fin de establecer las direcciones futuras de la empresa según sus prioridades competitivas (David, 2013) (Chase, Jacobs y Alquilano, 2014) (Boon-itt, Wong y Wong, 2017). 


\section{METODOLOGÍA}

Primera fase - Captura y análisis de los procesos de suministro, producción y distribución

El proyecto comenzó con el análisis de los procesos de suministro, producción y distribución para determinar las características del sector, recolectar datos relevantes e identificar oportunidades de mejora. Esta fase inició con la recolección de información secundaria y primaria en Puñizas, distrito de San Luis de Shuaro, Chanchamayo; con entrevistas a los productores y pobladores de la zona. Duró doce meses y el análisis se enfocó en la distribución de los flujos de los productores a los supermercados DC-12 y DC 10, de los productores a los terminales pesqueros de Villa María del Triunfo y Ventanilla y de los productores a los restaurantes de comida típica de la selva.

\section{Segunda fase - Evaluación con entrevistas de profundidad}

La segunda fase consistió en identificar características del mercado potencial, las necesidades de los clientes y la propuesta de valor del proyecto. Esta fase se caracterizó por las entrevistas a profundidad con preguntas abiertas, se entrevistaron a los jefes de pescadería y los gerentes de los supermercados DC-10 y DC-12, los responsables de compra de los restaurantes, expertos en el sector del Producción (Produce) y se realizaron entrevistas en los terminarles pequeros.

Los resultados de las entrevistas resaltaron la calidad, las propiedades organolépticas y las bondades en la cocina de la gamitana pero enfatizaron la variabilidad en la oferta del producto (tamaño, parámetros en la cadena de frío, contextura de la carne y su disponibilidad). Con los datos obtenidos se realizó la estimación de la demanda anual mediante un método de pronóstico causal.

Tercera fase - Reformulación con la redefinición de la estrategia y rediseño de la cadena de suministro

La tercera fase, la redefinición de la estrategia y el rediseño de la cadena de suministro, se obtuvo con el establecimiento del plan estratégico de Puñizas SAC del 2018 al 2021, la alineación de los planes funcionales y el establecimiento de los procesos del negocio. El plan estratégico de negocio es una herramienta para establecer en forma clara, precisa y sencilla la visión, el entorno competitivo, los objetivos y la estrategia del proyecto; sobre la base de los procesos para el desarrollo de las actividades
(Weinberger, 2009) y transversalmente la sostenibilidad del negocio (Marine Stewardship Council, 2016).

El rediseño de la cadena de suministro se sustenta en la alineación de sus objetivos generales y específicos, políticas y procesos con sus operaciones, con la estrategia de la organización (Perez-Franco, Sheffi y Caplice, 2016) y sus elementos: bienes, cultura y capacidades como elementos internos. El mapa conceptual final muestra la articulación de la estrategia de la organización y la consistencia interna en términos de compatibilidad, coherencia y sinergia.

\section{RESULTADOS}

\section{Supermercados DC 12}

Los supermercados DC 12 tienen tres proveedores de gamitana, Puñizas SAC y dos empresas ubicadas en la selva central y en Pucallpa; estas tienen un puesto de venta en el terminal pesquero de Villa María del Triunfo. La demanda semanal está entre los 100 a 150 kilos; salvo entre los meses de abril y junio cuando sube a 200 o 250 kilos. El abastecimiento es variable, con un precio de S/.14.90 por kilo y el formato recomendable es de 300 gramos para la venta de pescado entero fresco y de 500 a 800 gramos para la venta de filete.

La ventaja de la gamitana es su resistencia luego de la cosecha, tiene una ventana de consumo de 48 horas y no hay producto con características similares; sin embargo, la trucha y la tilapia tienen mejores costos y un abastecimiento constante. Los principales consumidores de gamitana están en los distritos de San Juan de Lurigancho, Rímac, Independencia y Surquillo.

\section{Supermercados DC 10}

Los supermercados DC 10 tienen dos proveedores de gamitana, ubicados en la selva central y Loreto. El producto se recibe en el terminal pesquero de Villa María del Triunfo. La demanda semanal es de 300 a 350 kilos, en abril sube a 1000 kilos y en mayo baja a 700 kilos. El precio es de S/.14.99 el kilo y su formato de comercialización es pescado entero fresco con 350 gramos, formato plato.

La gamitana se comercializa en todos los supermercados DC 10 con un volumen variable, por la demanda de los clientes; pero requiere trabajar en la promoción de sus características y bondades para incentivar su consumo. 
Tabla 1. Expectativas y opiniones de las empresas.

\begin{tabular}{|l|c|c|c|c|}
\cline { 2 - 4 } \multicolumn{1}{c|}{} & $\begin{array}{c}\text { Respuestas } \\
\text { válidas }\end{array}$ & Media & $\begin{array}{c}\text { Desviación } \\
\text { estándar }\end{array}$ & $\begin{array}{c}\text { Coeficiente de } \\
\text { variación }\end{array}$ \\
\hline $\begin{array}{l}\text { Asegurar que sus productos y operaciones no } \\
\text { dañen el medio ambiente }\end{array}$ & 4916 & 4,38 & 1,030 & 23,51 \\
\hline $\begin{array}{l}\text { Proveer productos y servicios de buena calidad al } \\
\text { menor costo posible }\end{array}$ & 4896 & 4,24 & 1,080 & 25,47 \\
\hline Ayudar a reducir la brecha entre ricos y pobres & 4832 & 3,77 & 1,340 & 35,54 \\
\hline
\end{tabular}

Fuente: Global Scan. (2015). Cuestionario radar

Tabla 2. Nivel de confianza en las instituciones.

\begin{tabular}{|l|c|c|c|c|}
\cline { 2 - 4 } \multicolumn{1}{c|}{} & \multicolumn{1}{c|}{$\begin{array}{c}\text { Respuestas } \\
\text { válidas }\end{array}$} & Media & $\begin{array}{c}\text { Desviación } \\
\text { estándar }\end{array}$ & $\begin{array}{c}\text { Coeficiente de } \\
\text { variación }\end{array}$ \\
\hline Gobierno nacional & 4945 & 2,88 & 0,913 & 31.70 \\
\hline Grandes empresas peruanas & 4886 & 2,61 & 0,835 & 31,99 \\
\hline Empresas globales operando en el Perú & 4776 & 2,78 & 0,846 & 30,43 \\
\hline
\end{tabular}

Fuente: Global Scan. (2015). Cuestionario radar

\section{Restaurantes}

Las entrevistas se realizaron en treinta y tres restaurantes de comida típica de la selva, con una duración de cuatro meses. De los resultados se puede resaltar: cinco restaurantes $(15 \%)$ compran la gamitana con una frecuencia de una vez a la semana, a un precio entre los S/. 10 a S/. 18 por kilo y el consumo está entre seis a treinta y ocho kilos; cuatro restaurantes $(12 \%)$ adquieren el producto una vez cada dos semanas, el precio de los proveedores oscila entre los S/. 8 a S/. 17 por kilo y el consumo semanal es de cinco a diez kilos; cuatro restaurantes $(12 \%)$ compran con poca frecuencia a un precio entre S/ 12 y S/ 30 por kilo con un consumo mensual de seis kilos. Un total de 20 restaurantes (61\%) no compran gamitana, principalmente por desconocimiento y por la variabilidad del producto.

\section{DISCUSIÓN}

La empresas tienen una preocupación permanente sobre los principios éticos y las regulaciones de sus actividades; tomando una postura responsable sobre el impacto de sus operaciones en la sociedad (Banco Mundial, 2016). Los resultados de la encuesta radar sobre responsabilidad social (Global Scan, 2015) muestran una preferencia por productos y operaciones que no dañen el medio ambiente, proveer productos de buena calidad al menor costo y en menor grado ayudar a reducir la brecha entre ricos y pobres (Ver Tabla 1). Los valores de la encuesta fueron 1 - no deben hacerse responsables, 3 - deben hacerse parcialmente responsable, 5deben hacerse completamente responsables.

En el contexto peruano, la media mayor a 4 muestra un alto nivel de responsabilidad de las empresas proveedoras en cuanto al impacto en el medio ambiente al menor costo posible. Estas respuestas tienen relación con el impacto de los casos a nivel mundial. En el caso de ayudar a reducir la brecha entre ricos y pobres, es una pregunta comparativa y tiene relación con las certificaciones de comercio justo y responsable.

Los resultados del cuestionario radar 2015 sobre el nivel de confianza en el gobierno, se muestran en la tabla 2. Las grandes empresas peruanas y las empresas globales con operaciones trabajando en el Perú tienen un nivel de confianza medio, los valores solicitados fueron 1 - confío mucho, 2 - confía algo, 3 - no confía mucho y 4 - no confía en absoluto.

Los resultados muestran una alarmante falta de confianza en las instituciones peruanas (Global Scan , 2016), este contexto para empresas pequeñas como Puñizas es preocupante. 


\section{CONCLUSIONES}

La gamitana fresca es un producto con un crecimiento atractivo de la demanda en la ciudad de Lima por la calidad de su carne, sus propiedades organolépticas y su versatilidad en la cocina, sin embargo, un factor por mejorar es la variabilidad del producto. El producto se oferta de manera irregular y su obtención con una calidad uniforme es una restricción para aumentar su flujo en la cadena de suministro. Otro factor importante a considerar es el bajo nivel de integración de la cadena de suministro; sus entidades, principalmente productor y consumidor, tienen un nivel bajo de coordinación.

Actualmente, Puñizas SAC está trabajando en el punto de equilibrio. La empresa debería tener veinte estanques, con una producción mensual de cuatro toneladas para tener una oferta constante del producto. El mercado demanda un pescado fresco de 300 gramos y una presentación de 800 gramos para los filetes. El enfoque de sostenibilidad es reconocido por el mercado, pero está sujeto a la entrega de un producto de calidad y constante.

La redefinición de la estrategia de Puñizas SAC ha permitido establecer el horizonte de crecimiento y desarrollo de la empresa; con enfoque en la diferenciación. Por las características del producto y de la empresa, este enfoque generaría su consolidación en el mercado. El enfoque en sostenibilidad aportaría en la consolidación en el mercado, pero se debe mejorar la comunicación de las bondades del producto, el estricto control de la calidad (análisis microbiológico, análisis químico), las certificaciones de inspección y auditoría del producto final. Los stakeholders podrían crear oportunidades en el mercado, mediante la comunicación de las fortalezas de la gamitana fresca y en filete.

El rediseño de su cadena de suministro presenta limitaciones el proceso de distribución del producto desde el productor al cliente. Los productos de la selva peruana tienen una sola vía comercial de comunicación al mercado de Lima, los caminos alternativos implican sobre costos adicionales, los cuales, están en el ámbito gubernamental. El estudio, por el lado de la empresa, muestra como un punto crítico la gestión de riesgos y la transparencia en la comunicación entre las entidades. Estos dos puntos críticos son importantes para establecer estrategias conjuntas en casos de alarma, como alguna variación en la oferta, riesgo alimentario, contaminación sanitaria, alguna eventualidad en el transporte, entre otros.
La metodología en el rediseño y la alineación de la cadena de suministro con la estrategia de la organización y sus elementos, consolida la articulación de la estrategia de la organización y su consistencia interna orientada a los clientes: supermercados y restaurantes; con énfasis en la integración de los procesos, comunicación entre las entidades, cooperación en casos de crisis y confianza entre las entidades; bajo una estrategia orientada al push o empuje y la personalización de la gamitana en la presentación en producto fresco y en filete.

\section{REFERENCIAS BIBLIOGRÁFICAS}

[1] Atún, J., Lozano, A., Alarcón, R., Granados, F. y Guarneros, L. (2010). The physical distribution of goods in megapolis: strategies for policies on the location of logistics facilities with the Central Region of Mexico. Social and Behavioral Sciences, 6130-6140.

[2] BancoMundial.(2016).¿Quéesresponsabilidad social empresaria? Washington D.C: Banco Mundial.

[3] Bijman, J., Kamphius, B.M., Wiersinga, R.C., Danse, M.G., Zhang, X.Y. y Hu, D. (2010). Linking small - scale farmers to modern retail chains. En J. Trienekens, J. Top, J. van der Vorst, y A. Beulens, Towards effective food chains (pág. 320). Wageningen: Wageningen Academic Publishers.

[4] Boon-itt, S., Wong, C. y Wong, C. (2017). Service supply chain management process capabilities: measurement development. International Journal of Production Economics, 1-11.

[5] CAF Banco de Desarrollo de América Latina. (2017). Perfil logístico de Amércia Latina 2015. Montevideo: CAF.

[6] Campos, L. (2015). El cultivo de la gamitana en latinoamérica. Iquitos.

[7] Chase, R., Jacobs, R. y Aquilano, N. (2014). Adminstración de operaciones. Producción y cadena de suministro. México D.F: McGraw Hill.

[8] Chopra, S. (2013). Administración de la cadena de suministro. México D.F.: Pearson Educación.

[9] Curonisy, Y. y Pastén, J. I. (2016). Plan de negocios para la comercialización de gamitana en supermercados y restaurantes de Lima. Universidad del Pacífico, Lima. 
[10] David, F. (2013). Estregia Competitiva. México D. F: Pearson.

[11] Fenucci, J. (1988). Manual para la cria de camarones peneidos. Brasilia: Organización de las Naciones Unidas para la Agricultura y Alimentación.

[12] Global Scan . (2016). Trust in Global Companies. Londrés: Global Scan .

[13] Global Scan. (2015). Cuestionario radar sobre RSC. Toronto: Global Scan .

[14] Harrison, A. y Van Hoek, R. (2011). Logistics management and strategy. Edimburgo: Pearson Education Limited.

[15] Heckmann, P., Shorten, D. y Engel, H. (2003). Supply Chain Management at 21. The Hard Road to Adulthood. Virginia: Booz Allen Hamilton.

[16] Instituto Nacional de Estadística e Informática . (2018). Instituto Nacional de Estadística e Informática. Recuperado de https://www.inei. gob.pe/

[17] Instituto Tecnológico Pesquero del Perú . (2009). Información nutricional sobre algunos peces comerciales de la amazonía peruana. Lima : Instituto Tecnológico Pesquero del Perú.

[18] Krajewski, L. J., Ritzman, L. P. y Malhotra, M. K. (2013). Administración de operaciones. PEARSON.

[19] Marine Stewardship Council. (2016). Seafood consumers put sustainability before price and brand. Londrés: Marine Stewardship Council.

[20] Martinez , C., Chávez, M. y Olvera, M. (1988). La nutrición y la alimentación en la acuicultura de latinoamérica. Una diagnosis. Roma: Organización de las Naciones Unidas para la Agricultura y la Alimentación. Recuperado el 2018

[21] Ministerio de Comercio y Turismo. (2016). Análisis integral de la cadena logística en el Perú. Lima: Ministerio de Comercio y Turismo.

[22] Ministerio de Comercio y Turismo. (2016). Análisis integral de logística en Perú - Cacao. Lima: Ministerio de Comercio y Turismo.

[23] Ministerio de Comercio y Turismo. (2016). Análisis integral de logística en Perú - Café. Lima: Ministerio de Comercio y Turismo.
[24] Ministerio de la Producción . (2011). Panorama de la acuicultura mundial, América Latina y el Caribe y en el Perú. Lima: Dirección General de Acuicultura .

[25] Ministerio de la Producción. (2012). Situación actual de la acuicultura en el Perú. Lima: Despacho viceministerial de pesquería.

[26] Ministerio de la Producción. (2013). Dirección de políticas y desarrollo pesquero. Lima: Ministerio de la Producción.

[27] Ministerio de la Producción. (2013). Estudio de mercado de la gamitana Colossoma macropomum en Lima Metropolitana. Lima: Ministerio de la Producción.

[28] Ministerio de la Producción. (2016). Anuario estadítico pesquero y acuícola 2015. Lima: Produce.

[29] Ministerio de la Producción. (2017). Manual de cultivo de la gamitana. Lima: FONDEPES.

[30] Ministerio de la Producción. (2018). Anuario estadístico pesquero y acuícola - 2018. Obtenido de Ministerio de la Producción.

[31] Ministerio de Transportes y Comunicaciones. (2015). Estudio para el mejoramiento de la logística de distribución urbana de mercancías en Lima metropolitana y el Callao. Lima: Teirlog Ingeniería.

[32] Ministerio de Transportes y Comunicaciones. (2018). Mapas viales. Recuperado de http:// www.mtc.gob.pe/

[33] Oliver , K. y Webber, M. (1982). Supply-chain management: logistics catches up with strategy. En P. Klaus y S. Müller, The Roots of Logistics (pág. 420). Nuremberg: Springer.

[34] Perez-Franco, R., Sheffi, Y. y Caplice, C. (2016). Rethink the supply chain as conceptual thinking. International Journal of Production Economics, 384-396.

[35] Sociedad Nacional de Pesquería. (2014). Pescado Santo. Lima: Sociedad Nacional de Pesquería.

[36] Weinberger, K. (2009). La estrategia. Lima: Nathan Associates.

[37] Wheelen, T. y Hunger, D. (2013). Administración estratégica. Bogotá: Pearson. 